ESAIM: PROCEEDINGS, January 2014, Vol. 44, p. 322-337

SMAI Groupe MAS - Journées MAS 2012 - Session thématique

\title{
A SURVEY OF SOME RECENT RESULTS ON RISK THEORY
}

\author{
Florin Avram ${ }^{1}$, Romain Biard ${ }^{2}$, Christophe Dutang ${ }^{3}$, Stéphane Loisel $^{4}$ and \\ LANDY RABEHASAINA ${ }^{5}$
}

\begin{abstract}
The goal of this paper is to give recent results in risk theory presented at the Conference "Journée MAS 2012" which took place in Clermont Ferrand. After a brief state of the art on ruin theory, we explore some particular aspects and recent results. One presents matrix exponential approximations of the ruin probability. Then we present asymptotics of the ruin probability based on mixing properties of the claims distribution. Finally, the multivariate case, motivated by reinsurance, is presented and some contemporary results (closed forms and asymptotics) are given.
\end{abstract}

\section{INTRODUCTION, MODEL, AND KNOWN RESULTS}

We consider the following problem motivated by actuarial issues. We suppose that the capital $\left\{R_{t}, t \geq 0\right\}$ of an insurance company is a process that satisfies

$$
R_{t}=R_{0}+c t-\sum_{i=1}^{N_{t}} X_{i}
$$

where parameters are interpreted as follows:

- $c$ is the premium income rate,

- $\left\{N_{t}, t \geq 0\right\}$ is a counting process, typically a Poisson process with intensity $\lambda>0$, which counts the number of incoming claims,

- $\left(X_{i}\right)_{i \in \mathbb{N}}$ is a family of i.i.d. non negative r.v.'s that model claim amounts,

- $R_{0} \geq 0$ is the initial reserve of the insurance company.

When $\left\{N_{t}, t \geq 0\right\}$ is a general renewal process then (1) is referred to the Sparre Andersen model. Historically, (1) was first studied when $N_{t}$ was a simple Poisson process by Cramer and Lundberg, see Lundberg, 1903 and Cramér, 1903]. Traditionally, independence is assumed between $\left\{N_{t}, t \geq 0\right\},\left(X_{i}\right)_{i \in \mathbb{N}}$ and $R_{0}$. The aim of

${ }^{1}$ Laboratoire de Mathématiques et de leurs Applications, UMR CNRS 5142, Université de Pau et des Pays de l'Adour, Avenue de l’Université - BP 1155, 64013 Pau Cedex, France. Email : Florin.Avram@univ-Pau.fr

2 Laboratoire de Mathématiques de Besançon, UMR CNRS 6623, Université de Franche Comté, 16 route de Gray, 25030 Besançon cédex, France. Email: romain.biard@univ-fcomte.fr

${ }^{3}$ Institut de Recherche Mathématique Avancée, UMR 7501, Université de Strasbourg et CNRS, 7 rue René Descartes, F-67000 Strasbourg; Université du Maine, Avenue Olivier Messiaen, F-72000 Le Mans. Email : christophe.dutang@univ-lemans.fr

${ }^{4}$ Université de Lyon, Université Lyon 1, Institut de Science Financière et d'Assurances, 50 avenue Tony Garnier, F-69007 Lyon. Email: loisel@univ-lyon1.fr

${ }^{5}$ Laboratoire de Mathématiques de Besançon, UMR CNRS 6623, Université de Franche Comté, 16 route de Gray, 25030 Besançon cédex, France. Email: lrabehas@univ-fcomte.fr

(C) EDP Sciences, SMAI 2013 
this paper is two fold. In the present section we will review basic results that aim at determining the distribution of the ruin time defined as

$$
\tau:=\inf \left\{t \geq 0 \mid R_{t}<0\right\}
$$

which consists in determining the following quantities:

- the cdf of $\tau$, i.e. the probability of ruin before the horizon $t$ for an initial reserve $R_{0}=u$ : $\psi(u, t):=$ $P_{u}[\tau \leq t]$

- the probability of eventual ruin starting from $R_{0}=u: \psi(u):=P_{u}[\tau<+\infty]=\lim _{t \rightarrow+\infty} \psi(u, t)$.

Usually, determining $\psi(u, t)$ turns out to be difficult, and one rather tries to find either explicit expressions of $\psi(u)$ or some asymptotic equivalent of $\psi(u)$ as $u \rightarrow+\infty$.

In Subsection 0.1 we recall basic results concerning the computation of the ruin probability and we give some known estimates of $\psi(u)$. In Subsection 0.2 we see how one can obtain results on the ruin probability when there is some dependence between claims or between inter-occurrence times.

In Section 1] a review as well as some new methods and ideas are given in order to get accurate approximations of $\psi(u)$.

In Section 2, we consider a particular model where claims admit some kind of correlation and we obtain asymptotics of $\psi(u)$.

Finally, in Section 3 we consider an extension of the model (1) motivated by insurance-reinsurance, and study the exit time out of quadrants of multivariate ruin processes.

\subsection{Expressions and asymptotics of $\psi(u, t)$ and $\psi(u)$}

Explicit expressions of $\psi(u)$ are available in the compound Poisson case, for some particular claim size distributions, when the net profit condition is satisfied (i.e. when the insurer receives more premium than what he has to pay in average). It can be proved that it satisfies the following integro-differential equation

$$
0=c \psi^{\prime}(u)-\lambda \psi(u)+\lambda \int_{0}^{\infty} \psi(u-z) d F(z)
$$

where $F($.$) denotes distribution of the claims. The most classical case is when claim amounts are exponentially$ distributed. Then (3) can be reduced to a simple first order differential equation, and $\psi(u)$ is of the form $A e^{-R u}$, with $A$ and $R$ positive. Explicit expressions of $\psi(u)$ are also available when the claim size distribution is a mixture of two exponential distributions, or more generally phase-type.

In the general light-tailed case, one knows that the ruin probability decays exponentially fast in the initial reserve. The exact formula for exponential claim amounts becomes both a bound and an asymptotic formula for the ruin probability: one has $\psi(u) \leq e^{-R u}$ for all $u \geq 0$ and there exists some $C>0$ such that

$$
\psi(u) \sim C e^{-R u}
$$

as $u \rightarrow+\infty$. Coefficient $R$ is called the Cramér-Lundberg adjustment coefficient, and corresponds to the positive solution of an equation involving the moment generating function of the claim size distribution (see Theorem 1 for more details). Following the approach of [Gerber, 1974], it is possible to link the Cramér-Lundberg adjustment coefficient $R$ with the risk aversion coefficient $a$. If one measures a random claim amount $X$ thanks to indifference pricing method (which means that the insurer does not show any preference between not insuring the risk and bearing the risk after receiving premium $\pi)$, with exponential utility function $u(x)=\left(1-e^{-a x}\right) / a$, the insurer would ask for premium

$$
\pi=\frac{1}{a} \ln \left(E\left(e^{a X}\right)\right)
$$

Gerber, 1974 notes that if the insurer determines the premium following this principle, then the CramérLundberg adjustment coefficient $R$ is identical to the risk aversion parameter $a$. Conversely, if the insurer wants the ruin probability to decrease exponentially fast, he can use indifference pricing principle with exponential 
utility function.

In the heavy-tailed case, ruin probabilities decay in a much slower way to zero and are typically power-tailed in the most classical cases. Finite-time ruin probabilities also feature power tails in numerous models, due to regular variation theory results. Let us recall two theorems that correspond to those two important cases.

Theorem 1 ( Rolski et al., 1999]). In the Sparre Andersen model, $\left(N_{t}\right)_{t \geq 0}$ is a renewal process where interoccurence times $T_{i}$ are $i . i . d$. as $T$. Let us assume the net profit condition $E(X)<c E(T)$. If claim amounts and inter-occurrence times have a m.g.f. $M_{X}, M_{T}$, then there exists a constant $C$ such that, for all $u \geq 0$

$$
\psi(u) \underset{u \rightarrow+\infty}{\sim} C e^{-\gamma u},
$$

where $\gamma$ is the positive root of $M_{X}(r) M_{T}(-r c)=1$.

Theorem 2 ( Embrechts \& Veraverbeke, 1982]). In the Sparre Andersen model, $\left(N_{t}\right)_{t \geq 0}$ is a renewal process where inter-occurrence times $T_{i}$ are i.i.d. as T. Let us assume the net profit condition $E(X)<c E(T)$. Let $F_{X, 0}(x)=\int_{0}^{x} \bar{F}_{X}(y) d y / E(X)$. If $F_{X}$ and $F_{X, 0}$ are sub-exponential, i.e. for $x \rightarrow+\infty, \overline{F^{* 2}}(x) / \bar{F}(x) \rightarrow 2$, then

$$
\psi(u) \underset{u \rightarrow+\infty}{\sim} \frac{1}{c E(T)-E(X)} \int_{u}^{+\infty} \bar{F}_{X}(y) d y
$$

In particular, $\psi(u) \underset{u \rightarrow+\infty}{\sim}(k / u)^{\alpha-1}$, for $X \sim \operatorname{Pareto}(k, \alpha)$.

One common point between finite-time and infinite-time ruin probabilities is that it is much easier to address them if the case where the initial reserve is zero. When $u=0$, the infinite time ruin probability only depends on the claim size distribution through its mathematical expectation. The finite-time ruin probability is easy to compute thanks to Takacs's ballot lemma argument. The ballot lemma gives for example the answer to the following question: given that F. Hollande gets $51.62 \%$ of votes, if one picks up ballots randomly, what is the probability that F. Hollande is always ahead of N. Sarkozy during the count? It also provides the answer to this other question: with $u=0$, given that the surplus at time $T$ is $x>0$, what is the probability that ruin has not occurred? The ballot lemma enables one to use only distributions of the aggregate claim amount at the terminal time when the initial surplus is zero.

In the case where $u>0$, one has to condition on the last instant before time horizon, if it exists, when the risk process is equal to zero. This enables one to get a recursive formula for $\psi(u, t)$, equivalent to the so-called Picard-Lefèvre formula. The latter (see [Picard \& Lefèvre, 1997]) is based on Appell polynomials and the link between the two approaches may be done thanks to pseudo-compound Poisson distributions (see Rullière \& Loisel, 2004]).

\subsection{Relaxing the independence assumption}

There are many different ways to relax the independence assumption between the different risks involved in the classical risk model. One may consider exchangeable claim amounts instead of i.i.d. ones. Some results are given in this case in Section 3. Another practical way to do this is to allow for some dependence between $X_{i}$ and $T_{i}$, where $T_{i}$ is the $i$-th inter-occurrence time, while keeping couples $\left(X_{i}, T_{i}\right)_{i \geq 1}$ i.i.d.. In that case, as ruin is the same as ruin at claim instants, one may study the random walk whose increments $c X_{i}-T_{i}$ are i.i.d., which is of course very useful. Some other works consider similar types of dependence between claim sizes and occurrence times (see Albrecher \& Boxma, 2004], Albrecher \& Teugels, 2006] and [Boudreault et al, 2006] among others). Another possibility is to allow for correlation between claim sizes, and to allow it to vary over time, in particular in cases of correlation crises. During those phenomena (like in the 2007-08) crisis, risks that are considered to be independent suddenly become extremely correlated. It is possible to obtain asymptotic 
expressions of the finite ruin probability with correlation crises, see [Biard et al., 2008]). One must not forget another kind of dependence, that comes from the fact that insurance companies may react to large past claims by increasing the premium income rate. This generates correlation between claim history and the premium income rate which becomes stochastic. It is possible to study asymptotic behavior of ruin probabilities when premium is computed according to Buhlmann-type credibility adjustments. One problem is that customers may leave the company and choose another insurer if the price of their contract becomes too high in comparison to other offered ones. A current research direction is to study the stability of insurance markets with strong competition between insurers, under different sets of assumptions (see [Dutang et al., 2012b]).

\section{MATRIX EXPONENTIAL APPROXIMATIONS OF THE RUIN PROBABILITY}

\subsection{Motivation}

It happens often in risk theory, queueing theory, mathematical finance, and other applied probability branches, that results are more explicit under the assumption that the density of the input data $X_{1}, X_{2}, \ldots, X_{I} \in \mathbb{R}_{+}$is a combination of exponentials whose exponents have negative real part.

This suggests to approximate the empirical measure of the data by a "sparse sum of exponentials"

$$
I^{-1} \sum_{i=1}^{I} \delta_{X_{i}}(d t) \approx\left(\sum_{k=0}^{K} w_{k} e^{-\mu_{k} t}\right) d t,
$$

where the coefficients $w_{i}$ may possibly depend on $t$ and be polynomials. Equivalently, this amounts to approximating the empirical Laplace transform by a rational function

$$
I^{-1} \sum_{i=0}^{I} e^{-s X_{i}} \approx \frac{\sum_{i=0}^{n-1} a_{i} s^{i}}{\sum_{i=0}^{n} b_{i} s^{i}}
$$

where $a_{0}, \ldots, a_{n-1}, b_{0}, \ldots, b_{n}$ are real coefficients. Furthermore, (4) is equivalent to assuming that the approximating density satisfies a linear ODE system with constant coefficients, or that it is representable in matrix exponential form.

Definition 3. a) A continuous density on $[0, \infty)$ is called of phase-type $P H(\boldsymbol{\alpha}, A)$ if

$$
f(t)=\boldsymbol{\alpha} e^{A t} \boldsymbol{a}, \quad \forall t \geq 0 \Leftrightarrow \hat{f}(s)=\boldsymbol{\alpha}(s I-A)^{-1} \boldsymbol{a}, \text { with }
$$

(1) $\boldsymbol{\alpha}$ a probability row vector, $\boldsymbol{a}=-A 1,1$ a column vector of 1 's, and

(2) A a subgenerator matrix, satisfying $A_{i j} \geq 0$ for $i \neq j, A \mathbf{1} \leq \mathbf{0}$.

b) When a representation (6) exists, but the components $\boldsymbol{\alpha}, A, \boldsymbol{a}$ are not as in part a) (and thus nonnegativity may not hold), $f(t)$ is called a matrix exponential function. If moreover $f(t) \geq 0, \forall t \geq 0$, it is called a matrix exponential density.

The problem of approximation by matrix exponential densities is a notoriously difficult classic of applied probability, with roots in works like Erlang, 1909 Cox, 1955. It is our purpose here to recall its status quo, including some recent applications to ruin theory (Avram et al., 2012 Dumitrescu et al., 2013].

Some historical landmarks. A general approach via the theory of Chebyshev systems is sketched in the monographs Krein \& Nudelman, 1977, Karlin \& Studden, 1966], but for a long while only the particular case of Laguerre-Erlang-Tricomi-Weeks expansions (involving a polynomial multiplied by an exponential) was applied in practice. The first specific results relating to general matrix exponential functions appeared only recently, in works like [O'Cinneide, 1990, O'Cinneide, 1991], and in the system engineering literature - see for example Anderson et al., 1996]. For an excellent reference of the status-quo on phase-type representations, see for example [Commault \& Mocanu, 2003] and references therein. 
Risk theory approximations. In the context of risk and queueing theory, some well-known matrixexponential approximations of the ruin probability density $f($.$) are the Renyi, De Vylder, Gamma, and Whitt-$ Ramsay approximations, obtained respectively by fitting one, two or three moments of the ruin time distribution $\tau$ (see Grandell, 2000 for a review).

In view of the scarcity of approximations fitting more than three moments, it is natural to ask the following question.

What are the difficulties blocking the development of high order moments based approximations of ruin probabilities?

\subsection{Padé approximations}

It turns out that all the approximations previously mentioned as well as their natural generalizations to higher order may be viewed as Padé or two-point Padé approximations of the Laplace transform of the ruin probability Avram et al., 2012] (given by the Pollaczek-Khinchine formula). As a consequence, implementations are available in most symbolic computing systems (which exploit the fact that the conversion from moments to a Padé approximation of the Laplace transform requires only solving a linear system). In Mathematica for example, the command is PadeApproximant.

\subsubsection{The admissibility of Padé approximations in probability}

While in principle a great tool due to their easiness of implementation, and their convergence for large $n$, Padé approximations (and generalizations, like multi-point Hermite-Padé interpolation) applied to Laplace transforms in probability have the drawback of the difficulty to guarantee "admissible inverses", i.e. nonnegative densities or non-increasing survival functions, when fitting three moments or more is desired (note that for fitting two moments $m_{1}, m_{2}$ of a nonnegative random variable, the Gamma approximation

$$
f(x) \sim \tilde{f}(x)=\frac{(\mu x)^{\alpha-1}}{\Gamma(\alpha)} \mu e^{-\mu x}, \alpha=\frac{m_{1}^{2}}{m_{2}-m_{1}^{2}}, \mu=\frac{m_{1}}{m_{2}-m_{1}^{2}}
$$

provides an easy admissible solution).

The question of admissible inverses is related to the positive realization problem in systems engineering; it is typically phrased in terms of a given rational Laplace transform, but we may equivalently suppose a given inverse, i.e. a given combination of exponentials.

Recently, several constructive algorithms have been proposed, like for example the one implemented in the CheckMEPositiveDensity command of the public domain package BUTools.

It is still an open problem to obtain minimal order representations and we will illustrate the difficulties involved in the next section by an example, restricting to the easier case of real exponents (which brings us to the realm of Chebyshev systems, i.e. systems for which combinations of $n$ terms may have at most $n-1$ roots).

\subsection{Problem 1: The nonnegativity of combinations of real exponentials}

We have to find conditions on the real weights $w_{k}, k=0,1, \ldots, d$ in terms of the given "exponents/frequencies" $0<\mu_{0}<\mu_{1}<\ldots<\mu_{d}$ so that the sum of exponentials with distinct exponents

$$
f(t)=\sum_{k=0}^{d} w_{k} e^{-\mu_{k} t}
$$

is nonnegative on $[0, \infty)$. Putting $x=e^{\left(-\mu_{1}+\mu_{0}\right) t} \in[0,1]$, one may rewrite this as

$$
f(t) e^{\mu_{0} t}=w_{0}+\sum_{k=1}^{d} w_{k} x^{\gamma_{k}}, \quad 1=\gamma_{1}<\gamma_{2}<\ldots<\gamma_{d}
$$


(and suppose w.l.o.g. $w_{0}=1$ ). This may therefore be also viewed as a problem of nonnegativity of Müntz polynomials on $[0,1]$.

One case in which nonnegativity is automatic is when one has obtained somehow a phase-type representation, but this is by no means an easy task.

To understand the difficulties involved, it is useful to consider an elementary example with three exponentials, where elementary calculus suffices [Dehon \& Latouche, 1982, Horv et al., 2009] (for two exponentials, the nonnegativity of $\tilde{f}(0)$ and nonnegativity of the coefficient of the asymptotically dominant exponent are clearly necessary and sufficient).

Example 4. Consider the following example due to Harris [Harris et al., 1992] (see also [Fackrell, 2003, Ch. 5.4])

$$
\begin{aligned}
& \bar{F}(t)=2 e^{-t}-3 e^{-2 t}+2 e^{-3 t} \Leftrightarrow \\
& f(t)=2 e^{-t}-6 e^{-2 t}+6 e^{-3 t}=6 e^{-t}\left(\frac{1}{3}-e^{-t}+e^{-2 t}\right)
\end{aligned}
$$

This is admissible, since the minimum of $g(t)=\frac{1}{3}-e^{-t}+e^{-2 t}$, obtained when $e^{-t^{*}}=\frac{1}{2}$, is $g\left(t^{*}\right)=\frac{1}{12}>0$.

Such explicit computations are impossible with more than three exponents; it becomes then interesting to look for phase-type representations

$$
f(t)=\boldsymbol{\alpha} e^{t A} \boldsymbol{a}, \boldsymbol{a}=(-A) \mathbf{1}
$$

with $A$ a Markovian generating matrix (this is trivial here, with A diagonal) and with $\boldsymbol{\alpha}$ a probability vector (the existence of such a pair $(\boldsymbol{\alpha}, A)$ immediately implies positivity).

(1) Because of the negative middle coefficient, our function is not "hyperexponential" (it is not phase-type representable by a diagonal matrix).

(2) The next thing to look for is a Coxian (ordered, bidiagonal) representations of order three, which may be obtained by a Newton/Cox partial fractions decomposition of the Laplace transform

$$
f^{*}(s)=\frac{2\left(s^{2}+2 s+3\right)}{(s+1)(s+2)(s+3)}=\frac{2}{3} \frac{6}{(s+1)(s+2)(s+3)}-\frac{1}{3} \frac{6}{(s+2)(s+3)}+\frac{2}{3} \frac{3}{s+3} .
$$

This yields a representation with

$$
A=\operatorname{bidiag}(-1,-2,-3)=\left(\begin{array}{lll}
-1 & 1 & 0 \\
0 & -2 & 2 \\
0 & 0 & -3
\end{array}\right)
$$

with nonpositive "Coxian coordinates"

$$
\left(\alpha_{1}, \alpha_{2}, \alpha_{3}\right)=(2 / 3,-1 / 3,2 / 3)
$$

implying that $f(t)$ is not representable as a Coxian density of order 3 .

(3) When A has real eigenvalues, if a phase-type representation exists, it is always possible to find a Coxian one [O'Cinneide, 1990], possibly of high order. A systematic way to detect the order is to look for

"Erlang majorizing extensions"

$$
\lambda e: \operatorname{Em} B=\left(\begin{array}{ccc}
A & \boldsymbol{a} & 0 \\
0 & -y & y \\
0 & 0 & -y
\end{array}\right)
$$

obtained by adding a single Erlang block $E_{n}(y)$ of "appropriate size $n$ " and frequency $y$ (for convenience we represented an Erlang block $E_{2}(y)$ ). 
One such representation of order 4 is:

$$
B_{4}=B=\left(\begin{array}{llll}
-1 & 1 & 0 & 0 \\
0 & -2 & 2 & 0 \\
0 & 0 & -3 & 3 \\
0 & 0 & 0 & -4
\end{array}\right)
$$

where the "extra eigenvalue" -4 may be obtained by the "extension formula" [Avram et al., 2012]:

$$
y=\frac{1}{2}\left(\mu_{3}+\mu_{2}+\sqrt{\left(\mu_{3}-\mu_{2}\right)^{2}+4 \mu_{1} \mu_{2} \frac{\alpha_{1}}{\alpha_{3}}}\right),
$$

Once a "Markovian majorizing" matrix $B$ is specified, it is easy to compute new "extended Coxian coordinates" by

$$
\beta=\alpha P=(1 / 2,0,0,1 / 2) .
$$

Since these are nonnegative, $B_{4}$ is a minimal order positive representation

The command CheckMEPositiveDensity of BUTools only obtains here a representation of order 5 , obtained by adding an Erlang(2) of frequency 6, illustrating the difficulty of the problem and the fact that further implementation improvements are possible.

Let us note that in risk theory it would furthermore be useful to have admissible symbolic approximations depending on important parameters like $\rho:=\psi(0)=\frac{\lambda m_{1}}{c}$, where $m_{1}$ is the first moment of the claims.

At second order, such approximations are not hard to obtain, as illustrated by the following new approximation for the ruin probabilities of the perturbed model from Avram et al., 2012.

\subsection{Second order approximations for the ruin probabilities of the Cramér Lundberg pro- cess with Brownian perturbation}

Let us consider the ruin process (1), to which a Brownian Motion $\{\sigma B(t), t \geq 0\}$ is added :

$$
R_{t}=R_{0}+c t-\sum_{i=1}^{N_{t}} X_{i}+\sigma B(t)
$$

The Laplace exponent of ruin process is given by

$$
\kappa(s)=p s+\frac{\kappa_{2}}{2} s^{2}+\sum_{k=3}^{\infty}(-s)^{k} \frac{\lambda m_{k}}{k !},
$$

where $m_{k}$ are the moments of the claims, $\kappa_{2}=\lambda m_{2}+\sigma^{2}$, and $p=c-\lambda m_{1}$.

Now we have two further unknowns of interest, the probability of "creeping ruin" $\psi_{d}$ (due to the diffusion) and that of "ruin by jump" $\psi_{j}$ (due to a claim). Their respective Laplace transforms satisfy

$$
\psi_{d}^{*}(s)=\frac{\sigma^{2} / 2}{\kappa^{\prime}(0)} \varphi(s)=\frac{\sigma^{2} / 2}{p} \varphi(s), \quad \psi_{j}^{*}(s)=\frac{1}{s}-\frac{\varphi(s)}{s}-\frac{\sigma^{2} / 2}{p} \varphi(s)
$$

where $\varphi(s):=1-s \psi^{*}(s)$.

We would like to produce Padé $(n-1, n)$ approximations, where the order $n$ is defined in (5). The Padé $(0,1)$ approximations are unreasonable, since they cannot satisfy the boundary conditions $\psi_{d}(0)=1, \psi_{j}(0)=0$. To satisfy those as well as the equation

$$
\psi_{d}^{*}(s)=\frac{\sigma^{2} / 2}{p} \varphi(s) \Leftrightarrow 1-s \psi_{j}^{*}(s)=\left(s+\frac{2 p}{\sigma^{2}}\right) \psi_{d}^{*}(s)
$$


which follows from (10), we must use at least a Padé $(1,2)$ approximation.

Theorem 5. Consider the exponential approximation

$$
\begin{aligned}
& \psi_{d}(x)=\frac{a_{d}-\mu_{1}}{\mu_{2}-\mu_{1}} e^{-\mu_{1} x}+\frac{\mu_{2}-a_{d}}{\mu_{2}-\mu_{1}} e^{-\mu_{2} x} \\
& \psi_{j}(x)=\frac{a_{j}}{\mu_{2}-\mu_{1}}\left[e^{-\mu_{1} x}-e^{-\mu_{2} x}\right]
\end{aligned}
$$

for the "creeping ruin" and "ruin by jump", which satisfy $\psi_{d}(0)=1, \psi_{j}(0)=0$. Then, by fitting the first two moments of the aggregate loss $L$, one is led to the following admissible approximation:

$$
a_{d}=\frac{3 m_{2}}{m_{3}}, a_{j}=a_{d} \frac{\lambda m_{2}}{\sigma^{2}}=\frac{3 \lambda m_{2}^{2}}{\sigma^{2} m_{3}} .
$$

and $-\mu_{1}$ and $-\mu_{2}$ the negative roots of $s^{2}+\left(a_{d}+a_{j}+\frac{2 p}{\sigma^{2}}\right) s+a_{d} \frac{2 p}{\sigma^{2}}=0$ (whose discriminant is non-negative).

Proof: $\psi^{*}(s), \psi_{d}^{*}(s)=\frac{s+a_{d}}{s^{2}+b_{1} s+b_{0}}$ are quotients of monic polynomials (to satisfy $\lim _{s \rightarrow \infty} s \psi_{d}^{*}(s)=\psi_{d}(0)=1$ ), with three free coefficients, but the second condition in 11 imposes one more condition $b_{0}=\frac{2 p}{\sigma^{2}} a_{d}$ (so that $\left.\psi_{j}^{*}(s)=\frac{a_{j}}{s^{2}+b_{1} s+a_{d} \frac{2 p}{\sigma^{2}}}\right)$, leaving only two free coefficients. Finally, fitting the first two coefficients around 0 of

$$
\varphi(s)=1-s \psi^{*}(s)=\frac{2 p}{\sigma^{2}} \psi_{d}^{*}(s) \approx \frac{2 p}{\sigma^{2}} \frac{s+a_{d}}{s^{2}+\left(a_{d}+a_{j}+\frac{2 p}{\sigma^{2}}\right) s+a_{d} \frac{2 p}{\sigma^{2}}}
$$

yields:

$$
\left\{\begin{array}{l}
\frac{\sigma^{2}}{2 p}\left(1+\frac{a_{j}}{a_{d}}\right)=\frac{\lambda m_{2}+\sigma^{2}}{2 p} \Leftrightarrow \frac{a_{j}}{a_{d}}=\frac{\lambda m_{2}}{\sigma^{2}} \\
\frac{\sigma^{2}}{2 p} \frac{a_{j}}{a_{d}^{2}}=\frac{\lambda m_{3}}{6 p}
\end{array}\right.
$$

with solution (14). Then, assuming $\mu_{1}<\mu_{2}$, Laplace inversion yields (12).

Moreover, both $\psi_{j}$ and $\psi_{d}$ are admissible. Indeed, this is obvious for $\psi_{j}$, since its initial value and its dominant coefficient $\frac{a_{j}}{\mu_{2}-\mu_{1}}$ are non-negative.

The same is true for $\psi_{d}$; indeed, we may check that its dominant coefficient is non-negative, i.e. that $\mu_{1} \leq a_{d}$, by noting that $s^{2}+\left(a_{d}+a_{j}+2 p / \sigma^{2}\right) s+a_{d} 2 p / \sigma^{2}$ is negative at $s=-a_{d}\left(\right.$ since $\left(a_{d}\right)^{2}+\left(a_{d}+a_{j}+2 p / \sigma^{2}\right)\left(-a_{d}\right)+$ $a_{d} 2 p / \sigma^{2}=-a_{d} a_{j}<0$, with $a_{d}$ and $a_{j}$ being positive). Therefore, $\mu_{1}<a_{d}<\mu_{2}$.

Remark 6. It is easy to check that this approximation is exact for exponential claims. Indeed, in that case the density transform in the parametrization (15) is:

$$
\begin{aligned}
& \varphi(s)=\frac{p}{\kappa(s)}=\frac{1}{1+\frac{\sigma^{2}}{2 p} s+\frac{\lambda}{p} s \frac{m_{1}}{s+m_{1}^{-1}}}=\frac{s+m_{1}^{-1}}{\left(s+m_{1}^{-1}\right)\left(1+\frac{\sigma^{2}}{2 p} s\right)+\frac{\lambda}{p} s m_{1}} \\
& =\frac{s+m_{1}^{-1}}{\frac{\sigma^{2}}{2 p} s^{2}+s\left(\frac{\lambda}{p} m_{1}+\frac{\sigma^{2}}{2 p} m_{1}^{-1}+1\right)+m_{1}^{-1}}=\frac{2 p}{\sigma^{2}} \frac{s+m_{1}^{-1}}{s^{2}+s\left(\frac{2 \lambda m_{1}}{\sigma^{2}}+m_{1}^{-1}+\frac{2 p}{\sigma^{2}}\right)+m_{1}^{-1} \frac{2 p}{\sigma^{2}}}
\end{aligned}
$$

with $a_{d}=1 / m_{1}, a_{j}=2 \lambda m_{1} / \sigma^{2}$, and it is easy to check that this coincides with our approximation [14], in the case of exponential claims.

\subsection{Problem 2 : Moments fitting and Hermite-Padé interpolation}

The matrix exponential/rational Laplace family may be viewed as an attempt to deal with the incomplete information inherent in large data sets via a finite "ergonomic" parametrization. For summarizing the data, one 
may use the empirical moments $m_{1}, m_{2}, \ldots, m_{K}$ (which are the coefficients of the power series expansion of the Laplace transform around 0), or, more generally, a finite number of values of the empirical Laplace transform $f^{*}(0), f^{*}\left(s_{1}\right), \ldots, f^{*}\left(s_{K}\right)$, sampled at "well-chosen" points like the Chebyshev points.

One fundamental open problem in the field, related to and generalizing the venerable Chebyshev-MarkovStieltjes moments problem, is to produce minimal or quasi-minimal matrix exponential approximations fitting exactly or approximatively a finite set of moments/values of the Laplace transform of a density. This is quite challenging already in the case of moments fitting, and currently only three moments exact fitting formulas are available Bobbio et al., 2005. (for more moments there is also an elegant approach of Johnson and Taaffe [Johnson \& Taaffe, 1989], which unfortunately produces approximations of about twice the minimal order in the case of three moments fitting).

We end by mentioning a new numeric approach for obtaining non-negativity certificates, based on upper and lower bounding of the exponentials by polynomials Dumitrescu et al., 2013. This approach allows optimizing matrix exponential approximations with respect to arbitrary convex objectives, and may turn out useful in investigating the open problems above.

\section{On an ASymptotic RUle $A+B / u$ For Ultimate RUin PROBabilities Under DEPENDENCE BY MIXING}

In this section, we focus on asymptotics of the ultimate ruin probability. For the Sparre Andersen model with light-tailed claim amounts, $\psi(u) \sim C e^{-\gamma u}$ as $u \rightarrow \infty$, see Theorem 2 With heavy-tailed claim amounts, the ruin probability is in the class of Hall of order 1 since $\psi(u) \sim C / u^{\alpha}$ as $u \rightarrow \infty$, see Theorem 1 In a recent paper, Albrecher et al., 2011] discussed the ruin probability when there is dependence by mixing in the claim amounts $\left(X_{i}\right)_{i \geq 1}$. They derived an asymptotic formula $\psi(u) \sim A+B / u$ as $u \rightarrow+\infty$ for Pareto correlated claims or inter-arrival times. We consider the same insurance context where the claim amounts $\left(X_{i}\right)_{i \geq 1}$ or the claim waiting times $\left(T_{i}\right)_{i \geq 1}$ are dependent through a mixing model. Such a form of dependence can translate some uncertainty on the model parameters due to incomplete available information. Another possible situation is when the model parameters are not univocally fixed but depend on heterogeneous socio-economic factors.

The free surplus of an insurance company at time $t$ is modeled by (1), where claim amounts $\left(X_{i}\right)_{i \geq 1}$ are still independent from the claim arrival process $\left(N_{t}\right)_{t>0}$. This time, however, we assume that the $X_{i}$ 's are i.i.d. random variables conditionally on a latent random variable $\Theta$ (given $\Theta=\theta$, they are distributed as $(X \mid \Theta=\theta)$ ). The variable $\Theta$ can be used to translate, for example, the uncertainty in the claim amounts or their heterogeneity. In practice, we consider a continuous distribution for $\Theta$, otherwise in case of discrete distribution, the ruin probability (16) decreases exponentially. Ruin occurs as soon as the surplus process becomes negative. Given $\Theta=\theta$, the ruin probability is thus defined as $\psi(u, \theta)=P\left(\exists t>0: U_{t}<0 \mid U_{0}=\right.$ $u, \Theta=\theta)$. For exponentially distributed claims, i.e. when $(X \mid \Theta=\theta) \sim \mathcal{E}(\theta)$, one gets the well-known formula $\psi(u, \theta)=\min \left(\frac{\lambda}{\theta c} e^{-u(\theta-\lambda / c)}, 1\right)$, where $\min (\ldots)<1$ under the net profit condition $\theta>\lambda / c \equiv \theta_{0}$. Integrating over the parameter $\theta$ yields for the (global) ruin probability

$$
\psi(u)=F_{\Theta}\left(\theta_{0}\right)+\int_{\theta_{0}}^{\infty} \frac{\theta_{0}}{\theta} e^{-u\left(\theta-\theta_{0}\right)} d F_{\Theta}(\theta)
$$

where $F_{\Theta}$ is the distribution function of $\Theta$. Note that (16) can also be interpreted from a regulator point-of-view. In a given territory and for a given policy, a regulator supervises a set of insurers facing exponentially distributed claim severities, but each insurer has its own parameters. In such a situation, the constant term $F_{\Theta}\left(\theta_{0}\right)$ is the proportion of insurers that will bankrupt irrespective of their initial capital $u$, while the $u$-dependent term $I\left(u, \theta_{0}\right)$ is the proportion of insurers that will bankrupt with a capital $u$. The distribution $\Theta$ is thus the heterogeneity among insurers.

In this context, the dependence between claim amounts is given by Property 2.1 of [Albrecher et al., 2011]. 
Proposition 7. When claim sizes fulfill for each $n \geq 1, P\left(X_{1}>x_{1}, \ldots, X_{n}>x_{n} \mid \Theta=\theta\right)=\prod_{i=1}^{n} \exp \left(-\theta x_{i}\right)$, then they have a dependence structure due to an Archimedean survival copula with generator $\phi=L_{\Theta}^{-1}$, the inverse Laplace transform of $\Theta$.

We now examine two particular distributions for the latent variable $\Theta$ that were reported in Albrecher et al., 2011. Firstly, we consider for $\Theta$ a gamma distribution $\mathcal{G} a(\alpha, \lambda)$ with density $f_{\Theta}(\theta)=\lambda^{\alpha} \theta^{\alpha-1} e^{-\lambda \theta} / \Gamma(\alpha)$, where $\Gamma($.$) denotes the gamma function; e.g. [Olver et al, 2010.]. The resulting claim amount X$ has a Pareto type II distribution with parameters $\alpha$ and $\lambda$, whose survival function is $P(X>x)=1 /(1+x / \lambda)^{\alpha}$ for $x \geq 0$. $>$ From (16), we then obtain the following known result.

Proposition 8. In the continuous time model with $(X \mid \Theta=\theta) \sim \mathcal{E}(\theta)$ and $\Theta \sim \mathcal{G} a(\alpha, \lambda)$,

$$
\psi(u)=\frac{\gamma\left(\alpha, \theta_{0} \lambda\right)}{\Gamma(\alpha)}+\frac{\lambda^{\alpha} \theta_{0}}{\Gamma(\alpha)} \frac{\Gamma\left(\alpha-1, \theta_{0}(\lambda+u)\right)}{(\lambda+u)^{\alpha-1}} e^{\theta_{0} u}
$$

provided $\alpha>1$.

Secondly, consider for $\Theta$ a stable $1 / 2$ distribution (also called a Lévy distribution $\mathcal{L} e(\alpha)$ ) with density (as defined in Albrecher et al., 2011]) $f_{\Theta}(\theta)=\alpha e^{-\alpha^{2} / 4 \theta} /\left(2 \sqrt{\pi \theta^{3}}\right)$. The resulting claim distribution is a Weibull distribution with parameters $1 / 2$ and $1 / \alpha^{2}$ whose tail distribution is $P(X>x)=e^{-\alpha \sqrt{x}}$ for $x \geq 0$. Using the generalized incomplete upper gamma function (see e.g. Chaudry \& Zubair, 2012]) leads us to the following known expression for the ruin probability:

Proposition 9. In the continuous time model with $(X \mid \Theta=\theta) \sim \mathcal{E}(\theta)$ and $\Theta \sim \mathcal{L} e(\alpha)$,

$$
\begin{aligned}
\psi(u)=\operatorname{erfc}\left(\frac{\alpha}{2 \sqrt{\theta_{0}}}\right)+\frac{\theta_{0} \sqrt{u}}{\alpha} e^{u \theta_{0}} & {\left[\left(1-\frac{1}{\alpha \sqrt{u}}\right) e^{\alpha \sqrt{u}} \operatorname{erfc}\left(d_{+}\right)\right.} \\
+ & \left.\left(1+\frac{1}{\alpha \sqrt{u}}\right) e^{-\alpha \sqrt{u}} \operatorname{erfc}\left(d_{-}\right)-\frac{2}{\sqrt{\pi u \theta_{0}}} e^{-u \theta_{0}-\alpha^{2} /\left(4 \theta_{0}\right)}\right],
\end{aligned}
$$

where $d_{+}=\sqrt{u \theta_{0}}+\alpha /\left(2 \sqrt{\theta_{0}}\right), d_{-}=\sqrt{u \theta_{0}}-\alpha /\left(2 \sqrt{\theta_{0}}\right)$ and erfc(.) denotes the complementary error function; e.g. Olver et al, 2010.].

These two propositions raise the question of how decrease the ruin probability (16) as a function of $u$. In Proposition 8 , one can hope to derive an asymptotic using an asymptotic expansion of the incomplete beta function. But in Proposition 9. we cannot obtain a valid expansion by using an asymptotic of the incomplete upper gamma function. Interestingly, a general result can be derived with (16) for a large class of distribution for $\Theta$.

Theorem 10. Consider the risk model with $(X \mid \Theta=\theta) \sim \mathcal{E}(\theta)$. Let $\theta_{0}=\lambda / c$. If $\Theta$ has a continuous distribution such that $f_{\Theta}$ is $C^{k-1}$ almost everywhere on $\left[\theta_{0},+\infty\left[\right.\right.$ and $f_{\Theta}^{(k)}$ is Lebesgue integrable on $\left[\theta_{0},+\infty\left[\right.\right.$ for $k \in \mathbb{N}^{\star}$, then

$$
\psi(u)=F_{\Theta}\left(\theta_{0}\right)+\sum_{i=0}^{k-1} \frac{h^{(i)}(0)}{u^{i+1}}+o\left(\frac{1}{u^{k}}\right)
$$

where $h(x)=\theta_{0} f_{\Theta}\left(x+\theta_{0}\right) /\left(x+\theta_{0}\right)$, so that

$$
h^{(i)}(0)=\sum_{j=0}^{i}(-1)^{j} \frac{i !}{(i-j) ! \theta_{0}^{j}} f_{\Theta}^{(i-j)}\left(\theta_{0}\right) .
$$

Remark 11. In the previous theorem with $k=1$ in item (ii), we get the $A+B / u$ rule $\psi(u)=F_{\Theta}\left(\theta_{0}\right)+\frac{f_{\Theta}\left(\theta_{0}\right)}{u}+$ $o\left(\frac{1}{u}\right)$, when $f_{\Theta}$ is almost everywhere differentiable on $\left[\theta_{0},+\infty\left[\right.\right.$ and $f_{\Theta}^{\prime}$ being a Lebesgue-integrable. 
Remark 12. A similar approach can be done when mixing the waiting times $\left(T_{1}, T_{2}, \ldots\right)$. Using Albrecher et al., 2011]'s Section 3, we have

$$
\psi(u)=\bar{F}_{\Lambda}\left(\lambda_{0}\right)+\int_{0}^{\lambda_{0}} \psi_{u}(\lambda) d F_{\Lambda}(\lambda), \text { with } \psi_{u}(\lambda)=\frac{\lambda}{\lambda_{0}} e^{-u / \theta\left(1-\lambda / \lambda_{0}\right)}, \lambda_{0}=\theta c .
$$

Under the same assumptions as Theorem 10 , we also obtain the $A+B / u$ rule. We give here only the first terms of the series expansion assuming that $\Lambda$ has a continuous distribution

$$
\psi(u)=\bar{F}_{\Lambda}\left(\lambda_{0}\right)+\frac{1}{c u} f_{\Lambda}\left(\lambda_{0}\right)+o\left(\frac{1}{u}\right) .
$$

Remark 13. In the discrete time framework, similar results can be derived when claim amounts have a zeromodified geometric distribution. See [Dutang et al., 2012a] for details.

For the present model by mixing, the survival function is the following Stieltjes integral $P(X>x)=$ $\int_{0}^{+\infty} e^{-\theta x} d F_{\Theta}(\theta)$, which is the Laplace transform of the random variable $\Theta$. Here too, one can hope that similar arguments as in Theorems 10 work.

Proposition 14. Let us consider the continuous time framework and assume $\Theta$ has a continuous distribution with density $f_{\Theta}$. If $f_{\Theta}$ is almost everywhere differentiable on $\mathbb{R}_{+}$with $f_{\Theta}^{\prime}$ being a Lebesgue-integrable, then for $x>0, P(X>x)=\frac{f_{\Theta}(0)}{x}+o\left(\frac{1}{x}\right)$. If $f_{\Theta}$ is $C^{\infty}$ in the neighborhood of the origin, then for $x>0$, $P(X>x) \underset{x \rightarrow+\infty}{\sim} \sum_{k=0}^{+\infty} \frac{f_{\Theta}^{(k)}(0)}{x^{k+1}}$.

Remark 15. The reason why the behavior of the integrand function $f_{\Theta}$ at the origin matters is explained by Laplace's method, which studies the asymptotics of the following integral $I(x)=\int_{a}^{b} e^{x p(t)} q(t) d t$, where $p$ and $q$ are continuous functions around $a$, the point assumed to be the minimum of $p$ in $[a, b[$. In our case, $p(t)=t$, hence the minimum of the exponent on $\mathbb{R}_{+}$is attained at the origin. See e.g. 2.3(iii) of [Olver et al, 2010.].

In this section, we present a class of dependent risk models, where the dependence is based on a mixing approach. We validate the $A+B / u$ rule suggested in Albrecher et al., 2011] for the ruin probability, which applies both when the claim amounts or the waiting times are correlated. Generally, even when some dependence is added in the claim arrival process, e.g., a Markovian setting, the decreasing shape of the ruin probability remains unchanged compared to the corresponding independent case: either exponential $e^{-\gamma u}$ or polynomial $u^{-\alpha}$. Hence, our particular mixing approach, leading to $A+B / u$ asymptotics, significantly worsens the situation for the insurer.

\section{Multivariate Ruin theory}

Ruin theory in dimension $N \geq 2$ is mainly motivated by the following problem: insurers often need the use of reinsurers as a means of safety to prevent them from extreme scenarions such as natural disasters, major earthquakes or unusually large (centenial) floods. A natural extension of (1) is to add one or more risk processes, i.e. to consider

$$
\left\{\begin{array}{l}
R_{t}^{1}=R_{0}^{1}+c_{1} t-S_{t}^{1} \\
R_{t}^{2}=R_{0}^{2}+c_{2} t-S_{t}^{2}
\end{array}\right.
$$

with deterministic initial reserves $R_{0}^{1}=x_{1}$ and $R_{0}^{2}=x_{2} .\left\{R_{t}^{1}, t \geq 0\right\}$ and $\left\{R_{t}^{2}, t \geq 0\right\}$ may be seen as the insurer and reinsurer's capital at time $t$. Processes $\left\{S_{t}^{i}, t \geq 0\right\}, i=1,2$ are correlated jump processes. Similarly to ruin time $\tau$ defined in (2), we let

$$
\begin{aligned}
\tau_{\text {or }} & :=\inf \left\{t \geq 0 \mid R_{t}^{1}<0 \text { or } R_{t}^{2}<0\right\} \\
\tau_{\text {sim }} & :=\inf \left\{t \geq 0 \mid R_{t}^{1}<0 \text { and } R_{t}^{2}<0\right\},
\end{aligned}
$$


respectively the ruin time of one of the processes, and of both processes. Likewise we let the probabilities of eventual ruin starting from $\left(R_{0}^{1}, R_{0}^{2}\right)=\left(x_{1}, x_{2}\right)$

$$
\begin{aligned}
\psi_{\mathrm{or}}\left(x_{1}, x_{2}, T\right) & :=P\left(\tau_{\mathrm{or}} \leq T \mid\left(R_{0}^{1}, R_{0}^{2}\right)=\left(x_{1}, x_{2}\right)\right), \\
\psi_{\text {sim }}\left(x_{1}, x_{2}, T\right) & :=P\left(\tau_{\text {sim }} \leq T \mid\left(R_{0}^{1}, R_{0}^{2}\right)=\left(x_{1}, x_{2}\right)\right) .
\end{aligned}
$$

The main difficulty comes from the fact that, because of correlation between the jump processes, processes $\left\{R_{t}^{1}, t \geq 0\right\}$ and $\left\{R_{t}^{2}, t \geq 0\right\}$ are correlated. For the general model, obtaining precise results concerning ruin probabilities 20 is difficult without imposing a particular structure on $S_{t}^{1}$ and $S_{t}^{2}$. In the following, two kinds of correlation will be studied:

- The case where $S_{t}^{1}=\alpha s(t), S_{t}^{2}=(1-\alpha) s(t)$ for some $\alpha \in(0,1)$ and some compound Poisson process $\{s(t), t \geq 0\}$. This corresponds to the situation where a fixed fraction of incoming claims are shared between the insurer and the reinsurer, also known as quota share.

- The case where $S_{t}^{1}$ and $S_{t}^{2}$ are issued from independent processes (again for example compound Poisson processes) and independent claims occurring according to a common Poisson process. This situation corresponds to "common shocks" in the bivariate process.

\subsection{Closed expressions}

\subsubsection{Integro differential equation}

As in the one dimensional case, a first approach for determining expressions of $\psi_{\text {or }}$ or $\psi_{\text {sim }}$ is to derive an integro differential equation akin to 3 . Letting $\hat{\psi}_{\text {or }}\left(x_{1}, x_{2}, \delta\right)=E_{\left(x_{1}, x_{2}\right)}\left(e^{-\delta \tau_{\text {or }}} \mathbb{1}_{\left\{\tau_{\text {or }}<+\infty\right\}}\right)$ the Laplace transform of the ruin probability, this here takes the following form when claims arrive according to a Poisson process with intensity $\lambda$ :

$$
\begin{aligned}
0=c_{1} \partial_{x_{1}} \hat{\psi}_{\mathrm{or}}\left(x_{1}, x_{2}, \delta\right)+c_{2} \partial_{x_{2}} & \hat{\psi}_{\mathrm{or}}\left(x_{1}, x_{2}, \delta\right) \\
& -(\lambda+\delta) \hat{\psi}_{\mathrm{or}}\left(x_{1}, x_{2}, \delta\right)+\lambda \int_{0}^{+\infty} \int_{0}^{+\infty} \hat{\psi}_{\mathrm{or}}\left(x_{1}-z_{1}, x_{2}-z_{2}, \delta\right) d F\left(z_{1}, z_{2}\right),
\end{aligned}
$$

accompanied by certain boundary conditions, and where $F(.,$.$) is the bivariate claims distribution. One has$ the same equation for $\psi_{\text {sim }}$, with different boundary conditions. We note that the equation corresponding to the probability of eventual ruin is obtained by taking $\delta=0$ in 21 .

Deriving 21 is often tedious and must be done with care, see e.g. Theorem 1 of [Avram et al, 2008b], Theorem 2.1 of [Dang et al, 2009] or Theorem 2.1 of [Hu \& Jiang, 2013], the latter for a model with interest rate. However the technical but major issues that arise are usually the following:

- One implicitly has to prove some differentiability properties for $\hat{\psi}_{\text {or }}(., ., \delta)$ on $(0,+\infty)^{2}$.

- Proper boundary conditions must be exhibited that completely charactarizes $\hat{\psi}_{\text {or }}(., ., \delta)$, i.e. such that uniqueness of the solution to 21 holds. Natural boundary conditions are the values for $\hat{\psi}_{\text {or }}\left(x_{1}, x_{2}, \delta\right)$ as $x_{1}$ or $x_{2}$ (possibly both) tend to infinity, however this is not the only option (see again Theorem 2.1 of $\mathrm{Hu} \&$ Jiang, 2013]).

Having addressed these issues, one then needs to solve Equation (21). Again, this depends a lot on the form of the claim processes $\left\{S_{t}^{1}, t \geq 0\right\}$ and $\left\{S_{t}^{2}, t \geq 0\right\}$. Dang et al Dang et al, 2009. opt for a recursive approach which is numerically interesting. Gong et al [Gong et al, 2012] also use a recursive approach, however without having to resort to use Equation 21.

\subsubsection{Geometrical considerations and reduction to simpler problems}

Another approach consists in reducing the two dimensional problem into several one dimensional first passage problems. This consists in exhibiting subsets of domain $(0,+\infty)^{2}$ that are transient or absorbing for process $\left\{\left(R_{t}^{1}, R_{t}^{2}\right), t \geq 0\right\}$. The problem is then about finding 
- the ruin time distribution starting from the absorbing set,

- the ruin time distribution starting from the transient set, conditioned not to enter the absorbing set in the meantime,

- the distribution of the entrance time into the absorbing set, given that no ruin occurs in the meantime.

Obtaining the three previous distributions may not look straightforward at first sight. However, things are less nasty than they seem, as the point is to find the absorbing and transient sets such that obtaining these distributions amount to solving easier one dimensional first passage problems. The quota share policy seems to be the most favorable situation when using this approach. In Avram et al, 2008a, Avram et al, 2008b Badescu et al, 2011, these sets are two cones. In Rabehasaina, 2009, these sets are two cones and one strip.

\subsection{Asymptotics}

As previously said, obtaining closed forms for $\psi_{\text {or }}$ or $\psi_{\text {sim }}$ is not always possible. Not only that, but expressions are sometimes hardly tractable and cannot be exploited numerically. An alternative is then to find the qualitative behavior of these quantities as the initial reserves $x_{1}$ and $x_{2}$ tend to infinity along a certain direction. The nature of the asymptotics depends a lot on the nature of the distribution of the claims. Two classes seem to stand out.

\subsubsection{Heavy tailed distributions}

In the case where claims admit a heavy tailed distribution then ruin probability of one of the branches $\psi_{\text {or }}$ verifies some asymptotics of the form

$$
\psi_{\mathrm{or}}\left(x_{1}, x_{2}, T\right) \propto T \bar{F}\left(x_{1}\right), \quad x_{1}, x_{2} \rightarrow+\infty,
$$

for some heavy tailed survival function $\bar{F}_{T}($.$) . We may cite two recent papers on this aspect that deal with$ the ruin probabilities in finite time. Biard considers the case of "common shocks" claims with regularly varying tailed claims distributions having a certain dependence structure:

Theorem 16 ( Biard, 2011]). Let us suppose that $\left(S_{t}^{1}, S_{t}^{2}\right)=\sum_{i=1}^{N_{t}}\left(X_{i}^{1}, X_{i}^{2}\right)$ where $\left(X_{i}^{1}, X_{i}^{2}\right)_{i \in \mathbb{N}}$ is i.i.d. of distribution $X^{i}=I^{i} W^{0}+\left(1-I^{i}\right) W^{i}$ for some independent r.v. $I^{1}, I^{2}, W^{j}, j=0,1,2$. $I^{1}$ and $I^{2}$ are $\mathcal{B}(p)$ distributed. The $W^{i}, i=0,1,2$, have regularly varying distribution with index $\alpha .\left\{N_{t}, t \geq 0\right\}$ is a Poisson process with intensity $\lambda$. We have

$$
\psi_{\text {or }}\left(x_{1}, \beta x_{1}, T\right) \sim C_{p} 2^{\alpha} \lambda T \bar{F}\left(x_{1}\right), \quad x_{1} \rightarrow \infty,
$$

where $C_{p}$ is an explicit constant depending only on $p$.

Recently, Hu and Jiang consider the case of quota share reinsurance with regularly varying distribution tailed claims and interest force:

Theorem 17 ( Hu \& Jiang, 2013). Let us suppose that $\left(S_{t}^{1}, S_{t}^{2}\right)=\left(\sum_{i=1}^{N_{t}} \sigma_{i}\right) \cdot\left(\delta_{1}, \delta_{2}\right)$ with $\delta_{1}+\delta_{2}=1$ and $\left(\sigma_{k}\right)_{k \in \mathbb{N}}$ i.i.d. claims having regularly varying tail with index $\alpha .\left\{N_{t}, t \geq 0\right\}$ is a Poisson process with intensity $\lambda$. Moreover, we suppose that risk processes are subject to a same interest rate $r>0$. Then

$$
\psi_{\text {or }}\left(x_{1}, x_{2}, T\right) \sim \lambda T \bar{F}_{T}\left(x_{2}\right), \quad x_{2} \geq \delta_{2} x_{1} / \delta_{1} \rightarrow+\infty,
$$

where $\bar{F}_{T}(x):=\frac{1}{T} \int_{0}^{T} P\left(e^{-r u} \sigma_{1}>x\right) d u$.

Note that the previous theorem deals with asymptotics with $x_{2} \geq \delta_{2} x_{1} / \delta_{1}$. When $x_{2}<\delta_{2} x_{1} / \delta_{1}$, the situation is much easier as, by a geometrical argument recalled in Hu \& Jiang, 2013 (stemming from Avram et al, 2008a) a reduction to a one dimension process can be made. 


\subsubsection{Light tailed distributions}

In that case, decay of the ruin probability is morally exponential or Weibull-type. Avram et al consider in (19) general spectrally negative Lévy processes for the risk processes $R_{t}^{1}$ and $R_{t}^{2}$, and establish the following asymptotics:

Theorem 18 ( Avram et al, 2008a $)$.

$$
\psi_{\text {or }}\left(x_{1}, \beta x_{1}\right) \sim C_{1} e^{-\gamma_{1} x_{1}}+C_{2} e^{-\gamma_{2} \beta x_{1}}, \quad x_{1} \rightarrow \infty
$$

where $C_{i}, i=1,2$, are explicit constants and $\gamma_{i}, i=1,2$, verify the Lundberg equation $1=E\left(e^{-\gamma_{i}\left(R_{1}^{i}-R_{0}^{i}\right)}\right)$.

Rabehasaina, 2012] also gives asymptotics of the form

$$
\frac{1}{x_{1}^{2-2 H}} \ln \psi_{\mathrm{or}}\left(x_{1}, x_{2}\right), \frac{1}{x_{1}^{2-2 H}} \ln \psi_{\operatorname{sim}}\left(x_{1}, x_{2}\right) \sim-C^{*}, \quad x_{1} \rightarrow+\infty, \quad x_{2} / x_{1}=\beta,
$$

for a model featuring a mix of claims occuring according to a compound Poisson process with light tailed claims and fractional brownian motion with Hurst parameter $H \in(1 / 2,1)$.

Acknowledgment: Stéphane Loisel acknowledges support from the research chair Actuariat Durable sponsored by Milliman Paris.

\section{REFERENCES}

[Albrecher et al., 2011]

[Albrecher \& Boxma, 2004]

[Albrecher \& Teugels, 2006]

[Anderson et al., 1996]

[Asmussen \& Albrecher, 2010]

[Avram et al., 2012]

[Dumitrescu et al., 2013]

[Avram et al, 2008a]

[Avram et al, 2008b]

[Badescu et al, 2011]

[Biard, 2011]

[Biard et al., 2008]

[Bobbio et al., 2005]

[Boudreault et al, 2006]
Albrecher, H., Constantinescu, C. \& Loisel, S. Explicit ruin formulas for models with dependence among risks. Insurance: Mathematics and Economics, 2011.

Albrecher, H. \& Boxma, O. A ruin model with dependence between claim sizes and claim intervals. Insurance: Mathematics and Economics, 35(2), pp.245-254, 2004.

Albrecher, H. \& Teugels, J.L. Exponential behavior in the presence of dependence in risk theory. Jounal of Applied Probability, 43(1), pp.257-273, 2006.

Anderson, B.D.O., Deistler, M., Farina, L. \& Benvenuti, L. Nonnegative realization of a linear system with nonnegative impulse response. Circuits and Systems I: Fundamental Theory and Applications, IEEE Transactions on, 43(2), pp.134-142, 1996.

Asmussen S. \& Albrecher, H., Ruin Probabilities, 2nd edition. World Scientific, 2010

Avram, F., Dumitrescu, B., Horvath, A., \& Pistorius, M.R. On matrix exponential approximations of the infimum of a spectrally negative Levy process. preprint (arXiv:1210.2611), 2012.

Dumitrescu, B., Sicleru, B., \& Avram, F. Semi-infinite optimization with sums of exponentials via polynomial approximation, preprint, 2013.

Avram, F., Palmowski, Z. \& Pistorius M. Exit problem of a two-dimensional risk process from the quadrant: Exact and asymptotic results. Annals of Applied Probability, 18(6), pp.2421-2449, 2008.

Avram, F., Palmowski, Z. \& Pistorius M. A two-dimensional ruin problem on the positive quadrant. Insurance: Mathematics and Economics, 42(1) (2008), pp.227-234, 2008.

Badescu, A.L., Cheung, E.C.K. \& Rabehasaina L. A two-dimensional risk model with proportional reinsurance. Journal of Applied Probability, 48(4), pp.749-765, 2011.

Biard, R. Asymptotic multivariate finite-time ruin probabilities with heavy-tailed claim amounts: Impact of dependence and optimal reserve allocation. preprint (http://hal.archives-ouvertes.fr/hal-00538571/fr/), 2011.

Biard, R., Lefèvre, C. \& Loisel, S. Impact of correlation crises in risk theory: asymptotics of finitetime ruin probabilities for heavy-tailed claim amounts when some independence and stationarity assumptions are relaxed. Insurance: Mathematics and Economics, 43(3), pp.412-421, 2008.

Bobbio, A., Horváth, A., \& Telek, M., Matching three moments with minimal acyclic phase type distributions. Stochastic models, 21(2-3), pp.303-326, 2005.

Boudreault, M., Cossette, H., Landriault, D. \& Marceau, E. On a risk model with dependence between interclaim arrivals and claim sizes. Scandinavian Actuarial Journal, 2006(5), pp.265-285, 2006 . 
[Chaudry \& Zubair, 2012]

[Commault \& Mocanu, 2003]

[Cox, 1955]

[Cramér, 1903]

[Dang et al, 2009]

[Dehon \& Latouche, 1982]

[Dutang et al., 2012a]

[Dutang et al., 2012b]

[Embrechts \& Veraverbeke, 1982]

[Erlang, 1909]

[Fackrell, 2003]

[Gerber, 1974]

[Gong et al, 2012]

[Grandell, 2000]

[Harris et al., 1992]

[Horv et al., 2009]

[Hu \& Jiang, 2013]

[Ignatov \& Kaishev, 2012]

[Johnson \& Taaffe, 1989]

[Karlin \& Studden, 1966]

[Krein \& Nudelman, 1977]

[Lefèvre \& Loisel, 2009]

[Lefèvre \& Picard, 2011]

[Lundberg, 1903]

[Nolan, 2012]

[Olver et al, 2010.]

[O'Cinneide, 1990]

[O'Cinneide, 1991]

[Picard \& Lefèvre, 1997]
Chaudry, M. A. \& Zubair, S. M. On a Class of Incomplete Gamma Functions with Applications. Chapman and Hall, 2012.

Commault, C. \& Mocanu, S. Phase-type distributions and representations: some results and open problems for system theory. International Journal of Control, 76(6), pp.566-580, 2003.

Cox, D.R. A use of complex probabilities in the theory of stochastic processes. Mathematical Proceedings of the Cambridge Philosophical Society,51, pp.313-319. Cambridge Univ Press, 1955. Cramér, H. On the mathematical theory of risk. Skandia Jubilee Volume, Stockholm 1903.

Dang, L., Zhu, N. \& Zhang H. Survival probability for a two-dimensional risk model. Insurance: Mathematics and Economics, 44, pp.491-496, 2009.

Dehon, M. \& Latouche, G. A geometric interpretation of the relations between the exponential and generalized Erlang distributions. Advances in Applied Probability, 14, pp. 885-897, 1982.

Dutang, C., Lefèvre, C. \& Loisel, S. On an asymptotic rule A+B/u for ultimate ruin probabilities under dependence by mixing. Submitted, 2012.

Dutang, C., Albrecher, H. \& Loisel, S. Competition among non-life insurers under solvency constraints: A game-theoretic approach. European Journal of Operational Research, 231 (3), pp. 702-711, 2013.

Embrechts, P. \& Veraverbeke, N. Estimates for the probability of ruin with special emphasis on the possibility of large claims. Insurance: Mathematics and Economics; 1(1), pp.55-72, 1982.

Erlang, A.K. The theory of probabilities and telephone conversations. Nyt Tidsskrift for Matematik B, 20:33-39, 1909.

Fackrell, M.W. Characterization of matrix-exponential distributions. PhD thesis, The University of Adelaide, 2003.

Gerber, H.U. On additive premium calculation principles. Astin Bulletin, 7(3), pp.215-222, 1974.

Gong, L., Badescu, A. \& Cheung, E.C.K. Recursive methods for a multi-dimensional risk process with common shocks. Insurance: Mathematics and Economics, 50(1), pp.109-120, 2012.

Grandell, J. Simple approximations of ruin probabilities. Insurance: Mathematics and Economics; 26, pp.157-173, 2000.

Harris, C.M., William, G.M. \& Robert, F.B. A note on generalized hyperexponential distributions. Stochastic Models, 8(1), pp.179-191, 1992.

Horváth, A., Rácz, S. \& Telek, M. Moments characterization of order 3 matrix exponential distributions. ASMTA '09 Proceedings of the 16th International Conference on Analytical and Stochastic Modeling Techniques and Applications, pp.174-188, 2009.

$\mathrm{Hu}$, Z.C. \& Jiang, B. On joint ruin probabilities of a two-dimensional risk model with constant interest rate. Journal of Applied Probability, 2013, to appear.

Ignatov, Z. G. and Kaishev, V. K. Finite time non-ruin probability for Erlang claim interarrivals and continuous interdependent claim amounts. Stochastics: an International Journal of Probability and Stochastic Processes, 84(4), pp.461-485, 2012.

Johnson M.A. \& M.R. Taaffe, M.R. Matching moments to phase distributions: Mixtures of Erlang distributions of common order. Stochastic Models, 5(4), pp.711-743, 1989.

S. Karlin and W.J. Studden. Tchebycheff systems: with applications in analysis and statistics, volume 376. Interscience Publishers New York, 1966.

MG Krein and AA Nudelman. The Markov moment problem and extremal problems, volume 50 of translations of mathematical monographs. American Mathematical Society, Providence, Rhode Island, 1977.

Lefèvre, C. and Loisel, S. Finite-time ruin probabilities for discrete, possibly dependent, claim severities. Methodology and Computing in Applied Probability, 11, pp.425-441, 2009.

Lefèvre, C. and Picard, P. A new look at the homogeneous risk model. Insurance: Mathematics and Economics, 49(3), pp.512-519, 2011.

Lundberg, F. I Approximerad fram ställning av sannolikhetsfunktionen, II Aterförsäkring av kollektivrisker. Almqvist \& Wiksell, Uppsala, 1903.

Nolan, J. P. Stable Distributions: Models for Heavy Tailed Data. Birkhauser, 2012.

Olver, F. W. J., Lozier, D. W., Boisvert, R. F. \& Clark, C. W. NIST Handbook of Mathematical Functions. Cambridge University Press, 2010.

O'Cinneide, C.A. Characterization of phase-type distributions. Stochastic Models, 6(1), pp.1-57, 1990 .

O'Cinneide, C.A. Phase-type distributions and invariant polytopes. Advances in Applied Probability, 23, pp.515-535, 1991.

Picard, P. \& Lefèvre, C. The Probability of Ruin in Finite Time with Discrete Claim Size Distribution. Scandinavian Actuarial Journal, 1997(1), pp.58-69, 1997. 
[Rabehasaina, 2009]

[Rabehasaina, 2012]

[Rolski et al., 1999]

[Rullière \& Loisel, 2004]
Rabehasaina, L. Risk processes with interest force in Markovian environment. Stochastic Models, 25(4), pp.580-613, 2009.

Rabehasaina, L. A Markov additive risk process in dimension 2 perturbed by a fractional Brownian motion. Stochastic Processes and their Applications, 122(8), pp.2925-2960, 2012.

Rolski, T., Schmidli, H., Schmidt, V. \& Teugels, J. Stochastic Processes for Insurance and Finance. Wiley Series in Proability and Statistics, 1999.

Rullière D. \& Loisel, S. Another look at the Picard-Lefèvre formula for finite-time ruin probabilities. Insurance: Mathematics and Economics, 35(2), pp.187-203, 2004. 